\title{
Radiation exposure from medical imaging: A silent harm?
}

\author{
Mathew Mercuri MSc, Tej Sheth MD, Madhu K. Natarajan MD MSc
}

See related research article by Eisenberg and colleagues, page 430

A dvances in medical imaging have had a considerable impact on medical practice over the past few decades. Regrettably, many such procedures expose patients to ionizing radiation, which may result in injury. Survey data show that physicians generally underestimate the magnitude of radiation doses and their associated effects, and thus underestimate the risk to patients undergoing medical imaging procedures. ${ }^{1-4}$ This view may be due to both the general lack of epidemiologic data specific to medical procedures and the nature with which radiation-induced injuries progress. In this issue of the journal, Eisenberg and colleagues help close the knowledge gap regarding the risk of cancer associated with exposure to low-dose ionizing radiation from medical imaging and therapeutic procedures in a contemporary cardiac population. ${ }^{5}$

As with all aspects of medical care, physicians must weigh the potential benefits against the potential harms when recommending a procedure using ionizing radiation. The benefits of such procedures tend to be evident. The risks, on the other hand, are not so apparent. The reason for this may be threefold.

First, in most cases it is difficult to predict who will be harmed, because many negative effects of exposure to ionizing radiation, such as cancer, are stochastic in nature (i.e., randomly occurring). However, although the severity of such effects does not vary by magnitude of exposure, the probability does increase with dose.

Second, the appearance or diagnosis of radiation-induced cancers can occur many years after exposure. Thus, it is difficult to attribute the cancer to a specific exposure.

Finally, much of what we know regarding cancer risk from ionizing radiation comes from large observational studies involving workers in the nuclear industry and atomic bomb survivors. Exposure in these groups is by and large much higher than those typically seen in clinical populations. Risks to individuals exposed to radiation at doses used in most medical imaging procedures must be extrapolated from epidemiologic data for "high-dose" populations (e.g., atomic bomb survivors) using a linear "no threshold" model ${ }^{6,7}$ However, there are no epidemiologic data to support the validity of this model for lowdose exposures $(<10$ millisieverts $[\mathrm{mSv}])$ and thus no direct data regarding the risk to individuals undergoing typical medical imaging procedures. $^{7}$ It is likely for these reasons that health care providers have difficulty quantifying the risks associated with many imaging procedures, because they have little or no epidemiologic data suitable to their patients or direct clinical experience with known radiation-induced injuries.

Common medical imaging tests, such as radiography and mammography, expose patients to radiation doses of less than $1 \mathrm{mSv}$. Assuming a linear no-threshold model, this level of exposure may not constitute a significant risk to individual patients. However, the frequency with which such tests are performed may pose a population risk. More sophisticated imaging modalities, such as computed tomography (CT), cardiac perfusion (nuclear stress) tests, and fluoroscopy-guided diagnostic and therapeutic procedures, often involve radiation doses in the range of 5-15 $\mathrm{mSv}$. These procedures fall in the low end of exposure for which we have epidemiologic evidence of increased cancer risk (i.e., acute dose of $>10 \mathrm{mSv})^{7}$ Again, this level of exposure may not pose much risk to the individual, but the risk may be important at the population level. The real trouble arises when an individual undergoes multiple tests or procedures.

As Eisenberg and colleagues show, a substantial number of patients underwent multiple procedures in a short period. ${ }^{5}$ A large observational study involving about one million adults (age 18$65)$ in the United States reiterates this point. ${ }^{8}$ Both
Competing interests: None declared.

This article was solicited and has not been peer reviewed.

Correspondence to: Mr. Mathew Mercuri, matmercuri@hotmail.com

CMAJ 2011. DOI:10.1503 /cmaj.101885 
studies show that multiple procedures in an individual can result in a cumulative exposure that approaches or exceeds the level for which there is reasonably good epidemiologic evidence of an increased risk of cancer (i.e., cumulative dose $>50 \mathrm{mSv}$ ). ${ }^{7}$ It is through multiple exposures that rather insignificant risks on an individual level can become significant for a particular patient.

The study by Eisenberg and colleagues shows a positive dose-response relation for radiation exposure and cancer risk at cumulative doses for which there is little previous epidemiologic evidence of increased cancer risk (i.e., cumulative dose $<50 \mathrm{mSv}){ }^{5}$ Despite the demonstrated risk, only a few of the cancers diagnosed in their study were likely a direct result of exposure to radiation from the medical imaging. For example, if we consider a lifetime risk of cancer of about $2.5 \%$ for every $1000-\mathrm{mSv}$ dose among individuals between the ages of 40 and 60 , we would expect one new cancer for every 2000 patients receiving a $20-\mathrm{mSv}$ dose that is directly attributable to the radiation exposure from the medical procedure. ${ }^{9}$ Many of the patients in the study by Eisenberg and colleagues received a dose in this range. However, such estimates are theoretical, based on extrapolations of risk at higher doses (assuming a linear projection of risk). Although the risk of cancer at this exposure level seems to be relatively small, the fact that a large number of individuals are undergoing multiple procedures does pose a potential risk at the population level.

The current wisdom for minimizing the risk to the patient is to adopt the ALARA (as low as reasonably achievable) principle. ${ }^{10}$ This principle can be realized in a number of ways. The first is through technological innovations, which may provide means of producing suitable images with reduced levels of radiation exposure. For example, innovations in cardiac CT technology over the past five years have resulted in a decrease in the minimum achievable dose from 10-16 $\mathrm{mSv}$ to as low as $1-3 \mathrm{mSv}$. Another way is for physicians to optimize their technique or protocols.

As with other health risks, the best strategy may be prevention. This could mean referring the patient for a procedure that involves lower or no radiation exposure or, in some circumstances, avoiding the procedure altogether. Prevention may become of prime importance in situations where a patient has undergone or is expected to undergo a series of tests or procedures with moderate to high levels of exposure to radiation. The standard by which one chooses to avoid a procedure or suggest an alternative may depend on whether the procedure is indicated for the purpose of improving prognosis or for diagnosis only. For example, in the case of primary angioplasty in acute myocardial infarction, the immediate and substantial improvement in prognosis may sufficiently outweigh the comparatively small risk of cancer in the long term. Communication between the referring physician and the radiologist or specialist should be encouraged in an effort to find the optimal diagnostic or therapeutic strategy for the patient.

It is not uncommon for a patient to be referred for a variety of medical imaging tests and procedures by different physicians. It can be difficult, therefore, for an individual physician to keep track of the patient's cumulative exposure (assuming information about the radiation dose is available for each procedure - it is often not).

The implementation of a strategy to track radiation doses may help physicians and patients stay aware of the cumulative exposure. Health care and nuclear industries currently use such strategies to monitor exposure among employees. However, as Rehani and Frush point out, there are currently no examples of good nationwide programs to do so for patients. ${ }^{11}$ Programs to track radiation doses, such as the International Atomic Energy Agency's Smart Card/SmartRadTrack Project (http://rpop.iaea.org/RPOP/RPoP/Content /News/smart-card-project.htm), are now becoming a reality. It is imperative that Canadian health care providers adopt a similar strategy.

\section{References}

1. Shiralkar S, Rennie A, Snow M, et al. Doctors' knowledge of radiation exposure: questionnaire study. BMJ 2003;327:371-2.

2. Krille L, Hammer GP, Merzenich H, et al. Systematic review on physician's knowledge about radiation doses and radiation risks of computed tomography. Eur J Radiol 2010;76:36-41.

3. Correia MJ, Hellies A, Andreassi MG, et al. Lack of radiological awareness among physicians working in a tertiary-care cardiological centre. Int J Cardiol 2005;103:307-11.

4. Rehani MM. Training of interventional cardiologists in radiation protection - the IAEA's initiatives. Int J Cardiol 2007;114: 256-60.

5. Eisenberg MJ, Afilalo J, Lawler PR, et al. Cancer risk related to low-dose ionizing radiation from cardiac imaging in patients following acute myocardial infarction. CMAJ 2011;183:430-6.

6. Lin EC. Radiation risk from medical imaging. Mayo Clin Proc 2010;85:1142-6.

7. Brenner DJ, Doll R, Goodhead DT, et al. Cancer risks attributable to low doses of ionizing radiation: assessing what we really know. Proc Natl Acad Sci U S A 2003;100:13761-6.

8. Fazel R, Krumholz HM, Wang Y, et al. Exposure to low-dose ionizing radiation from medical imaging procedures. $N$ Engl J Med 2009;361:849-57.

9. Vijayalakshmi K, Kelly D, Chapple CL, et al. Cardiac catheterization: radiation doses and lifetime risk of malignancy. Heart 2007;93:370-1.

10. 1990 recommendations of the International Commission on Radiological Protection. Ann ICRP 1991;21:1-201.

11. Rehani M, Frush D. Tracking radiation exposure of patients. Lancet 2010;376:754-5.

Affiliations: All of the authors are with the Division of Cardiology, Department of Medicine, Hamilton Health Sciences and McMaster University, Hamilton, Ont.

Contributors: Mathew Mercuri drafted the article, and Tej Sheth and Madhu Natarajan revised it for important intellectual content. All of the authors approved the final version of the manuscript submitted for publication. 